\title{
EULER-CESÀRO DIFFERENCE SPACES OF BOUNDED, CONVERGENT AND NULL SEQUENCES
}

\author{
FEYZI BAŞAR AND NAIM L. BRAHA
}

\begin{abstract}
In this paper, we introduce the spaces $\breve{\ell}_{\infty}, \breve{c}$ and $\breve{c}_{0}$ of Euler-Cesàro bounded, convergent and null difference sequences and prove that the inclusions $\ell_{\infty} \subset \breve{\ell}_{\infty}, c \subset \breve{c}$ and $c_{0} \subset \breve{c}_{0}$ strictly hold. We show that the spaces $\breve{c}_{0}$ and $\breve{c}$ turn out to be the separable BK spaces such that $\breve{c}$ does not possess any of the following: AK property and monotonicity. We determine the alpha-, beta- and gamma-duals of the new spaces and characterize the matrix classes $\left(\breve{c}: \ell_{\infty}\right),(\breve{c}: c)$ and $\left(\breve{c}: c_{0}\right)$.
\end{abstract}

\section{Introduction}

By $\mathbb{N}$ and $\mathbb{C}$, we denote the sets of all natural and complex numbers, respectively. Let $\omega$ be the vector space of all complex sequences. Any vector subspace of $\omega$ is called a sequence space. By $\ell_{\infty}, c, c_{0}$ and $\ell_{p}$; we denote the classes of all bounded, convergent, null and $p$-absolutely summable sequences, as usual; respectively. Moreover, we write $b s, c s$ and $c s_{0}$ to denote the spaces of all bounded, convergent and null series, respectively.

A sequence $\left(y_{n}\right)_{n=0}^{\infty}$ in a normed space $X$ is called a Schauder basis for $X$ if for every $x \in X$ there is a unique sequence $\left(\alpha_{n}\right)_{n=0}^{\infty}$ of scalars such that $x=\sum_{n=0}^{\infty} \alpha_{n} y_{n}$, i.e., $\left\|x-\sum_{k=0}^{n} \alpha_{n} y_{n}\right\| \rightarrow$ 0 , as $n \rightarrow \infty$. The alpha-, beta- and gamma-duals $\lambda^{\alpha}, \lambda^{\beta}$ and $\lambda^{\gamma}$ of a sequence space $\lambda$ are respectively defined by

$$
\begin{aligned}
& \lambda^{\alpha}:=\left\{a=\left(a_{k}\right) \in \omega: a x=\left(a_{k} x_{k}\right) \in \ell_{1} \text { for all } x=\left(x_{k}\right) \in \lambda\right\}, \\
& \lambda^{\beta}:=\left\{a=\left(a_{k}\right) \in \omega: a x=\left(a_{k} x_{k}\right) \in c s \text { for all } x=\left(x_{k}\right) \in \lambda\right\}, \\
& \lambda^{\gamma}:=\left\{a=\left(a_{k}\right) \in \omega: a x=\left(a_{k} x_{k}\right) \in b s \text { for all } x=\left(x_{k}\right) \in \lambda\right\} .
\end{aligned}
$$

Also, we use the conventions that $e=(1,1,1, \ldots)$ and $e^{(k)}$ is the sequence whose only non-zero term is 1 in the $k^{\text {th }}$ place for each $k \in \mathbb{N}$.

Received November 10, 2015, accepted February 19, 2016.

2010 Mathematics Subject Classification. 40C05, 40A05, 46A45.

Key words and phrases. Composition of summability methods, Cesàro mean of order one, Euler mean of order one, backward difference operator, sequence space, BK space, Schauder basis, Köthe-Toeplitz duals, matrix transformations.

Corresponding author: Naim L. Braha. 
Let $\lambda$ and $\mu$ be two sequence spaces, and $A=\left(a_{n k}\right)$ be an infinite matrix of complex numbers $a_{n k}$, where $n, k \in \mathbb{N}$. Then, we say that $A$ defines a matrix transformation from $\lambda$ into $\mu$ and we denote it by writing $A: \lambda \rightarrow \mu$, if for every sequence $x=\left(x_{k}\right) \in \lambda$ the $A$-transform $A x=\left\{(A x)_{n}\right\}$ of $x$ is in $\mu$; where

$$
(A x)_{n}=\sum_{k=0}^{\infty} a_{n k} x_{k} \text { for each } n \in \mathbb{N} .
$$

By $(\lambda: \mu)$, we denote the class of all matrices $A$ such that $A: \lambda \rightarrow \mu$. Thus, $A \in(\lambda: \mu)$ if and only if the series on the right side of (1.1) converges for each $n \in \mathbb{N}$, i.e., $A_{n} \in \lambda^{\beta}$ for all $n \in \mathbb{N}$ and every $x \in \lambda$, and we have $A x \in \mu$ for all $x \in \lambda$, where $A_{n}$ denotes the sequence in the $n$-th row of $A$.

The matrix domain $\lambda_{A}$ of an infinite matrix $A$ in a sequence space $\lambda$ is defined by

$$
\lambda_{A}:=\left\{x=\left(x_{k}\right) \in \omega: A x \in \lambda\right\}
$$

which is a sequence space. By using the matrix domain of a triangle matrix, so many sequence spaces have recently been defined by several authors, see for instance $[1,2,3,4,5,6,7,8,9,10$, $11,12,13,14,15]$. In the literature, the matrix domain $\lambda_{\Delta}$ is called the difference sequence space whenever $\lambda$ is a normed or a paranormed sequence space, where $\Delta$ denotes the backward difference matrix $\Delta=\left(\Delta_{n k}\right)$ and $\Delta^{\prime}=\left(\Delta_{n k}^{\prime}\right)$ denotes the transpose of the matrix $\Delta$, the forward difference matrix, which are defined by

$$
\begin{aligned}
& \Delta_{n k}=\left\{\begin{array}{cl}
(-1)^{n-k}, & n-1 \leq k \leq n, \\
0 & , 0 \leq k<n-1 \text { or } k>n,
\end{array}\right. \\
& \Delta_{n k}^{\prime}=\left\{\begin{array}{cl}
(-1)^{n-k}, & n \leq k \leq n+1, \\
0 & , 0 \leq k<n \text { or } k>n+1
\end{array}\right.
\end{aligned}
$$

for all $k, n \in \mathbb{N}$; respectively. The notion of difference sequence spaces was introduced by Kızmaz [16], who defined the sequence spaces

$$
X(\Delta):=\left\{x=\left(x_{k}\right) \in \omega: \Delta^{\prime} x=\left(x_{k}-x_{k+1}\right) \in X\right\}
$$

for $X \in\left\{\ell_{\infty}, c, c_{0}\right\}$. The difference space $b v_{p}$, consisting of all sequences $x=\left(x_{k}\right)$ such that $\Delta x=\left(x_{k}-x_{k-1}\right)$ is in the sequence space $\ell_{p}$, was studied in the case $0<p<1$ by Altay and Başar [4] and in the case $1 \leq p \leq \infty$ by Başar and Altay [17], and Çolak et al. [5]. Kirişçi and Başar [18] have introduced and studied the generalized difference sequence space

$$
\widehat{X}:=\left\{x=\left(x_{k}\right) \in \omega: B(r, s) x \in X\right\},
$$

where $X$ denotes any of the spaces $\ell_{\infty}, c, c_{0}$ and $\ell_{p}$ with $1 \leq p<\infty$, and $B(r, s) x=\left(s x_{k-1}+r x_{k}\right)$ with $r, s \in \mathbb{R} \backslash\{0\}$. Following Kirişçi and Başar [4], Sönmez [19] have been examined the sequence space $X(B)$ as the set of all sequences whose $B(r, s, t)$-transforms are in the space 
$X \in\left\{\ell_{\infty}, c, c_{0}, \ell_{p}\right\}$, where $B(r, s, t)$ denotes the triple band matrix $B(r, s, t)=\left\{b_{n k}(r, s, t)\right\}$ defined by

$$
b_{n k}(r, s, t)=\left\{\begin{array}{l}
r, n=k \\
s, n=k+1 \\
t, n=k+2 \\
0, \text { otherwise }
\end{array}\right.
$$

for all $k, n \in \mathbb{N}$ and $r, s, t \in \mathbb{R} \backslash\{0\}$. Also in [6, 7, 8, 9, 10, 11], certain difference sequence spaces are studied. Quite recently, Başar has studied the space $\widetilde{\ell}_{p}$ of $p$-absolutely $\widetilde{B}$-summable sequences, in [12].

In this paper, as a natural continuation of Başar [12], we introduce the spaces $\breve{\ell}_{\infty}, \breve{c}$ and $\breve{c}_{0}$ of Euler-Cesàro bounded, convergent and null difference sequences by using the composition of the Euler mean $E_{1}$ and Cesàro mean $C_{1}$ of order one with backward difference operator $\Delta$. The rest of this paper is organized, as follows: In Section 2, we give some required notations and basic concepts. In Section 3, we introduce the sequence spaces $\breve{\ell}_{\infty}, \breve{c}$ and $\breve{c}_{0}$, and establish some inclusion relations. Also, we construct the bases of the spaces $\breve{c}$ and $\breve{c}_{0}$. In Section 4 , the alpha-, beta-, gamma-duals of the spaces $\breve{\ell}_{\infty}, \breve{c}$ and $\breve{c}_{0}$ are determined and the classes $\left(\breve{c}: \ell_{\infty}\right),(\breve{c}: c)$ and $\left(\breve{c}: c_{0}\right)$ of matrix transformations are characterized. In the final section of the paper, we give a table for the literature related to the domain of certain triangle matrices on the spaces $c_{0}, c$ and $\ell_{\infty}$, and record some further suggestions.

\section{Preliminaries}

A $B$-space is a complete normed space. A topological sequence space in which all coordinate functionals $\pi_{k}$ with $\pi_{k}(x)=x_{k}$, are continuous is called a $K$-space. A BK-space is defined as a $K$-space which is also a $B$-space, that is, a $B K$-space is a Banach space with continuous coordinates. For example, the space $\ell_{p}$ is $B K$-space with $\|x\|_{p}=\left(\sum_{k=0}^{\infty}\left|x_{k}\right|^{p}\right)^{1 / p}$ and $c_{0}, c$ and $\ell_{\infty}$ are $B K$-spaces with $\|x\|_{\infty}=\sup _{k \in \mathbb{N}}\left|x_{k}\right|$, where $1 \leq p<\infty$. The sequence space $X$ is said to be solid (cf. [20, p. 48]) if and only if

$$
\widetilde{X}:=\left\{\left(u_{k}\right) \in \omega: \exists\left(x_{k}\right) \in X \text { such that }\left|u_{k}\right| \leq\left|x_{k}\right| \text { for all } k \in \mathbb{N}\right\} \subset X .
$$

Let us define the Euler mean $E_{1}=\left(e_{n k}\right)$ of order one and Cesàro mean $C_{1}=\left(c_{n k}\right)$ of order one by

$$
e_{n k}:=\left\{\begin{array}{l}
\frac{\left(\begin{array}{l}
n \\
k
\end{array}\right)}{2^{n}}, 0 \leq k \leq n, \\
0, k>n,
\end{array} \quad c_{n k}:=\left\{\begin{array}{c}
\frac{1}{n+1}, 0 \leq k \leq n, \\
0, k>n,
\end{array}\right.\right.
$$

for all $k, n \in \mathbb{N}$. Their inverses $E_{1}^{-1}=\left(g_{n k}\right)$ and $C_{1}^{-1}=\left(h_{n k}\right)$ are given by

$$
g_{n k}:=\left\{\begin{array}{cl}
\left(\begin{array}{l}
n \\
k
\end{array}\right)(-1)^{n-k} 2^{k}, & 0 \leq k \leq n, \\
0 & , k>n,
\end{array} \quad h_{n k}:=\left\{\begin{array}{cl}
(-1)^{n-k}(k+1), & n-1 \leq k \leq n, \\
0 \quad, 0 \leq k \leq n-2 \text { or } k>n
\end{array}\right.\right.
$$


for all $k, n \in \mathbb{N}$. We define the matrix $\widetilde{B}=\left(\widetilde{b}_{n k}\right)$ by the composition of the matrices $E_{1}, C_{1}$ and $\Delta$ as

$$
\widetilde{b}_{n k}:=\left\{\begin{array}{cl}
\frac{\left(\begin{array}{l}
n \\
k
\end{array}\right)}{2^{n}(k+1)}, & 0 \leq k \leq n \\
0 & , k>n
\end{array}\right.
$$

for all $k, n \in \mathbb{N}$.

\section{The Euler-Cesàro difference spaces of null, convergent and bounded sequences}

In this section, we define the spaces $\breve{c}_{0}, \breve{c}$ and $\breve{\ell}_{\infty}$ of Euler-Cesàro null, Euler-Cesàro convergent and Euler-Cesàro bounded difference sequences. Also, we present some inclusion theorems and construct the Schauder bases of the spaces $\breve{c}_{0}$ and $\breve{c}$.

Now, we introduce the spaces $\breve{c}_{0}, \breve{c}$ and $\breve{\ell}_{\infty}$ of Euler-Cesàro null, Euler-Cesàro convergent and Euler-Cesàro bounded difference sequences as the set of all sequences whose $\widetilde{B}$ transforms are in the spaces $c_{0}, c$ and $\ell_{\infty}$, respectively, i.e.,

$$
\begin{aligned}
\breve{c}_{0} & :=\left\{x=\left(x_{n}\right) \in \omega: \lim _{n \rightarrow \infty} \sum_{k=0}^{n} \frac{\left(\begin{array}{l}
n \\
k
\end{array}\right)}{2^{n}(k+1)} x_{k}=0\right\}, \\
\breve{c} & :=\left\{x=\left(x_{n}\right) \in \omega: \exists l \in \mathbb{C} \text { such that } \lim _{n \rightarrow \infty} \sum_{k=0}^{n} \frac{\left(\begin{array}{l}
n \\
k
\end{array}\right)}{2^{n}(k+1)} x_{k}=l\right\}, \\
\breve{\ell}_{\infty} & :=\left\{x=\left(x_{n}\right) \in \omega: \sup _{n \in \mathbb{N}}\left|\sum_{k=0}^{n} \frac{\left(\begin{array}{l}
n \\
k
\end{array}\right)}{2^{n}(k+1)} x_{k}\right|<\infty\right\} .
\end{aligned}
$$

With the notation of (1.2), the spaces $\breve{c}_{0}, \breve{c}$ and $\breve{\ell}_{\infty}$ can be redefined as follows:

$$
\breve{c}_{0}=\left(c_{0}\right)_{\widetilde{B}}, \quad \breve{c}=c_{\widetilde{B}} \text { and } \breve{\ell}_{\infty}=\left(\ell_{\infty}\right)_{\widetilde{B}}
$$

Define the sequence $y=\left(y_{n}\right)$ by the $\widetilde{B}$-transform of a sequence $x=\left(x_{k}\right)$, i.e.,

$$
y_{n}=(\widetilde{B} x)_{n}=\sum_{k=0}^{n} \frac{\left(\begin{array}{l}
n \\
k
\end{array}\right)}{2^{n}(k+1)} x_{k} \text { for each } n \in \mathbb{N} .
$$

Throughout the text, we suppose that the sequences $x=\left(x_{k}\right)$ and $y=\left(y_{k}\right)$ are connected with the relation (3.1). One can obtain by a straightforward calculation from (3.1) that

$$
x_{n}=\left(\widetilde{B}^{-1} y\right)_{n}=\sum_{k=0}^{n} \sum_{j=k}^{n}\left(\begin{array}{l}
j \\
k
\end{array}\right)(-1)^{j-k} 2^{k}(2 j-k+1) y_{k} \text { for each } n \in \mathbb{N} .
$$

The relation (3.2) gives that the inverse $\widetilde{B}^{-1}=\left(d_{n k}\right)$ of $\widetilde{B}$ in (2.1) is defined by

$$
d_{n k}:=\left\{\begin{array}{cc}
\sum_{j=k}^{n}\left(\begin{array}{l}
j \\
k
\end{array}\right)(-1)^{j-k} 2^{k}(2 j-k+1) & , 0 \leq k \leq n, \\
0 & , k>n
\end{array}\right.
$$

for all $k, n \in \mathbb{N}$. Here and after, by $\lambda$ we denote any of the sets $\breve{c}_{0}, \breve{c}$ and $\breve{\ell}_{\infty}$. 
Theorem 3.1. The set $\lambda$ is a linear space with the co-ordinatewise addition and scalar multiplication which is the BK-space with the norm $\|x\|_{\lambda}=\|\widetilde{B} x\|_{\infty}$.

Proof. Proof of the first part of the theorem is a routine verification and so, we omit the detail. Since $c_{0}, c$ and $\ell_{\infty}$ are the $B K$-spaces with respect to their usual sup-norm and $\widetilde{B}$ is a triangle matrix, Theorem 4.3.2 of Wilansky [21, p. 61] gives the fact that $\breve{c}_{0}, \breve{c}$ and $\breve{\ell}_{\infty}$ are the $B K$-spaces. This completes the proof.

Remark 3.2. One can easily check that the absolute property does not hold on the space $\lambda$, that is $\|x\|_{\lambda} \neq\||x|\|_{\lambda}$ for at least one sequence in the space $\lambda$, and this says that $\lambda$ is a sequence space of non-absolute type, where $|x|=\left(\left|x_{k}\right|\right)$.

Throughout the text $\mu$ denotes any of the spaces $c_{0}, c$ or $\ell_{\infty}$. With the notation of (3.1), since the transformation $T$ defined from $\lambda$ to $\mu$ by $x \mapsto y=T x$ is a linear bijection, we have the following:

Corollary 3.3. The sequence space $\lambda$ is linearly norm isomorphic to the space $\mu$.

Now, we give some inclusion relations concerning with the spaces $\lambda$ and $\mu$.

Theorem 3.4. The inclusion $\mu \subset \lambda$ strictly holds.

Proof. Let $x=\left(x_{k}\right) \in \mu$. Then, since it is immediate that

$$
\begin{aligned}
\|x\|_{\lambda}=\|\widetilde{B} x\|_{\infty} & =\sup _{n \in \mathbb{N}}\left|\sum_{k=0}^{n} \frac{\left(\begin{array}{l}
n \\
k
\end{array}\right)}{2^{n}(k+1)} x_{k}\right| \\
& \leq\|x\|_{\infty} \sup _{n \in \mathbb{N}} \sum_{k=0}^{n} \frac{\left(\begin{array}{l}
n \\
k
\end{array}\right)}{2^{n}(k+1)} \\
& =\|x\|_{\infty} \sup _{n \in \mathbb{N}} \frac{2^{n+1}-1}{2^{n}(n+1)}<\infty,
\end{aligned}
$$

the inclusion $\mu \subset \lambda$ holds. Now, consider the sequences $u=\left(u_{k}\right)=\left\{\left[1+(-1)^{k+1}\right] / 2\right\}_{k \in \mathbb{N}}$ and

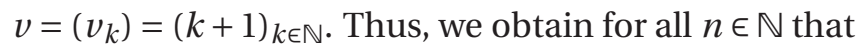

$$
\begin{aligned}
(\widetilde{B} u)_{n} & =\frac{1}{2^{n}} \sum_{k=0}^{n} \frac{\left(\begin{array}{l}
n \\
k
\end{array}\right)\left[1+(-1)^{k+1}\right]}{2(k+1)} \\
& =\frac{1}{2^{n+1}}\left[\sum_{k=0}^{n} \frac{\left(\begin{array}{l}
n \\
k
\end{array}\right)}{k+1}+\sum_{k=0}^{n} \frac{\left(\begin{array}{l}
n \\
k
\end{array}\right)(-1)^{k+1}}{k+1}\right] \\
& =\frac{1}{2^{n+1}}\left(\frac{2^{n+1}-1}{n+1}+\frac{n}{n+1}-1\right) \\
(\widetilde{B} v)_{n} & =\frac{1}{2^{n}} \sum_{k=0}^{n} \frac{\left(\begin{array}{l}
n \\
k
\end{array}\right)(k+1)}{k+1}=1 .
\end{aligned}
$$


Therefore, since $\widetilde{B} u \in c_{0}, u \in \breve{c}_{0}$ while $u \notin c_{0}$ and $\widetilde{B} v \in c \subset \ell_{\infty}, v$ is both in $\breve{c}$ and hence in $\breve{\ell}_{\infty}$ but $v$ does not neither in $c$ nor in $\ell_{\infty}$. This shows that the inclusions $c_{0} \subset \breve{c}_{0}, c \subset \breve{c}$ and $\ell_{\infty} \subset \breve{\ell}_{\infty}$ are strict.

One can see from Theorem 2.3 of Jarrah and Malkowsky [22] that the domain $v_{T}$ of an infinite matrix $T=\left(t_{n k}\right)$ in a normed sequence space $v$ has a basis if and only if $v$ has a basis, if $T$ is a triangle. As an immediate consequence of this fact, we have the following:

Corollary 3.5. Let $\alpha_{n}=(\widetilde{B} x)_{n}$ for all $n \in \mathbb{N}$. Define the sequence $\left\{u^{(n)}\right\}=\left\{u_{k}^{(n)}\right\}_{k \in \mathbb{N}}$ in the space $\breve{c}_{0}$ by

$$
u_{k}^{(n)}=\left\{\begin{array}{cl}
\sum_{j=k}^{n}\left(\begin{array}{l}
j \\
k
\end{array}\right)(-1)^{j-k} 2^{k}(2 j-k+1) & , 0 \leq k \leq n, \\
0 & , k>n
\end{array}\right.
$$

for every fixed $n \in \mathbb{N}$. Then, the following statements hold:

(a) The sequence $\left\{u^{(n)}\right\}_{n \in \mathbb{N}}$ is a basis for the space $\breve{c}_{0}$ and any $x \in \breve{c}_{0}$ has a unique representation of the form $x=\sum_{n=0}^{\infty} \alpha_{n} u^{(n)}$.

(b) The set $\left\{e, u^{(n)}\right\}$ is a basis for the space $\breve{c}$ and any $x \in \breve{c}$ has a unique representation of the form $x=l e+\sum_{n=0}^{\infty}\left(\alpha_{n}-l\right) u^{(n)}$, where $l=\lim _{k \rightarrow \infty}(\widetilde{B} x)_{k}$.

Since the sequence spaces $\breve{c}_{0}$ and $\breve{c}$ have Schauder bases, Corollary 3.5 directly gives the following:

Corollary 3.6. The sequence spaces $\breve{c}_{0}$ and $\breve{c}$ are separable.

Theorem 3.7. The sequence space $\breve{c}$ has not the AK property.

Proof. Let $x=(1,2,3,4, \ldots, n, \ldots) \in \breve{c}$. Then, the $n$th section $x^{[n]}$ of $x$ is $x^{[n]}=(1,2, \ldots, n, 0,0, \ldots)$. Therefore, we have

$$
\left\|x-x^{[n]}\right\|_{\breve{c}}=\sup _{k \geq n} \frac{1}{2^{k}}\left|\sum_{j=0}^{k}\left(\begin{array}{l}
k \\
j
\end{array}\right) \frac{1}{j+1}[(j+1)-0]\right|=\sup _{k \geq n} \frac{1}{2^{k}} \cdot 2^{k}=1 .
$$

In the sequel, we mention some concepts (see [23]) related to the sequence spaces.

A sequence space $\lambda$ is said to be symmetric if $x_{\sigma(n)} \in \lambda$ whenever $x \in v$, where $\sigma$ is any permutation in $\mathbb{N}$. A sequence algebra $v$ (see Maddox [24, p. 153]) is a linear space together with an internal operation of multiplication of elements of $v$ such that

$$
x y \in v, x(y z)=(x y) z, x(y+z)=x y+x z, \quad(x+y) z=x z+y z
$$

and

$$
\alpha(x y)=x(\alpha y) \text { for all scalars } \alpha \text {. }
$$

Now, we show some of the above properties for the sequence space $\breve{c}$. 
Theorem 3.8. The sequence space $\breve{c}$ is not monotone nor sequence algebra.

Proof. To prove the first part, we consider the sequences $x=\left(x_{n}\right)=(n+1)_{n \in \mathbb{N}} \in \breve{c}$ and $y=$ $\left(y_{n}\right)=\left\{(n+1)^{2}\right\}_{n \in \mathbb{N}}$. After some calculations it follows that $y \notin \breve{c}$ which proves that $\breve{c}$ is not monotone. To prove that $\breve{c}$ is not convergence-free we define the sequences: $x=\left(x_{n}\right)=(n+$ $1)=\left(y_{n}\right)=y$. Then, $x=y \in \breve{c}$, but $x y=\left(1^{2}, 2^{2}, 3^{2}, \ldots\right) \notin \breve{c}$. Really after some calculations, we get that

$$
\begin{aligned}
\lim _{n \rightarrow \infty} \frac{1}{2^{n}} \sum_{k=0}^{n}\left(\begin{array}{l}
n \\
k
\end{array}\right) \frac{1}{k+1}(k+1)^{2} & =\lim _{n \rightarrow \infty}\left[\frac{1}{2^{n}} \sum_{k=0}^{n}\left(\begin{array}{l}
n \\
k
\end{array}\right) k+\frac{1}{2^{n}} \sum_{k=0}^{n}\left(\begin{array}{l}
n \\
k
\end{array}\right)\right] \\
& =\lim _{n \rightarrow \infty}\left(\frac{n \cdot 2^{n-1}}{2^{n}}+1\right)=\infty .
\end{aligned}
$$

Hence, $x y \notin \breve{c}$.

\section{The alpha-, beta- and gamma-duals of the spaces $\breve{\ell}_{\infty}, \breve{c}$ and $\breve{c}_{0}$}

In this section, we determine the alpha-, beta- and gamma-duals of the spaces $\breve{\ell}_{\infty}, \breve{c}$ and $\breve{c}_{0}$ of Euler-Cesàro bounded, convergent and null difference sequences.

Now, we start with the following lemma due to Steieglitz and Tietz [25] which is needed in proving our theorems. Here and after, we denote the collection of all finite subsets of $\mathbb{N}$ by $\mathscr{F}$.

Lemma 4.1. Let $A=\left(a_{n k}\right)$ be an infinite matrix over the complex field. Then, the following statements hold:

(a) $A \in\left(c_{0}: \ell_{1}\right)=\left(c: \ell_{1}\right)=\left(\ell_{\infty}: \ell_{1}\right)$ if and only if

$$
\sup _{K \in \mathscr{F}} \sum_{n=0}^{\infty}\left|\sum_{k \in K} a_{n k}\right|<\infty .
$$

(b) $A \in\left(c_{0}: \ell_{\infty}\right)=\left(c: \ell_{\infty}\right)=\left(\ell_{\infty}: \ell_{\infty}\right)$ if and only if

$$
\sup _{n \in \mathbb{N}} \sum_{k=0}^{\infty}\left|a_{n k}\right|<\infty .
$$

(c) $A \in(c: c)$ if and only if (4.2) holds, and

$$
\begin{aligned}
& \exists\left(\alpha_{k}\right) \in \omega \text { such that } \lim _{n \rightarrow \infty} a_{n k}=\alpha_{k} \text { for all } k \in \mathbb{N}, \\
& \exists \alpha \in \mathbb{C} \text { such that } \lim _{n \rightarrow \infty} \sum_{k=0}^{\infty} a_{n k}=\alpha .
\end{aligned}
$$


Theorem 4.2. The alpha-dual of the spaces $\breve{\ell}_{\infty}, \breve{c}$ and $\breve{c}_{0}$ is the set

$$
d_{1}:=\left\{a=\left(a_{n}\right) \in \omega: \sup _{K \in \mathscr{F}} \sum_{n=0}^{\infty}\left|\sum_{k \in K} \sum_{j=k}^{n}\left(\begin{array}{l}
j \\
k
\end{array}\right)(-1)^{j-k} 2^{k}(2 j-k+1) a_{n}\right|<\infty\right\} .
$$

Proof. Let us define the matrix $M=\left(m_{n k}\right)$ via $a=\left(a_{n}\right) \in \omega$ by

$$
m_{n k}=\left\{\begin{array}{cc}
\sum_{j=k}^{n}\left(\begin{array}{l}
j \\
k
\end{array}\right)(-1)^{j-k} 2^{k}(2 j-k+1) a_{n}, & 0 \leq k \leq n, \\
0 & , k>n
\end{array}\right.
$$

for all $k, n \in \mathbb{N}$. Since the relation (3.2) holds, one can immediately derive that

$$
a_{n} x_{n}=\sum_{k=0}^{n} \sum_{j=k}^{n}\left(\begin{array}{l}
j \\
k
\end{array}\right)(-1)^{j-k} 2^{k}(2 j-k+1) a_{n} y_{k}=(M y)_{n}
$$

for all $n \in \mathbb{N}$. Therefore, we conclude by (4.3) that $a=\left(a_{n}\right) \in \lambda^{\alpha}$ if and only if $M \in\left(\mu: \ell_{1}\right)$. Then, we derive by Part (a) of Lemma 4.1 that

$$
\sup _{K \in \mathscr{F}} \sum_{n=0}^{\infty}\left|\sum_{k \in K} \sum_{j=k}^{n}\left(\begin{array}{l}
j \\
k
\end{array}\right)(-1)^{j-k} 2^{k}(2 j-k+1) a_{n}\right|<\infty
$$

which leads to the desired result that $\lambda^{\alpha}=d_{1}$.

Theorem 4.3. Define the set $d_{2}$ by

$$
d_{2}=\left\{a=\left(a_{i}\right) \in \omega:\left(\sum_{k=0}^{n} \sum_{i=k}^{n} \sum_{j=k}^{i}\left(\begin{array}{l}
j \\
k
\end{array}\right)(-1)^{j-k} 2^{k}(2 j-k+1) a_{i}\right) \in c\right\} .
$$

Then, $(\breve{c})^{\beta}=d_{2}$.

Proof. Define the triangles $\Delta^{-1}=S=\left(s_{n k}\right)$ and $B(a)=\left(b_{n k}\right)$ by

$$
s_{n k}=\left\{\begin{array}{l}
1,0 \leq k \leq n, \\
0, k>n,
\end{array} \quad b_{n k}=\left\{\begin{array}{c}
a_{n} d_{n k}, 0 \leq k \leq n, \\
0, k>n
\end{array}\right.\right.
$$

for all $k, n \in \mathbb{N}$; where $a=\left(a_{n}\right) \in \omega$ and $d_{n k}$ is defined as in (3.3). Let $\widetilde{A}=\left(\widetilde{a}_{n k}\right)$ be the composition of the matrices $S$ and $B(a)$, i.e.,

$$
\widetilde{a}_{n k}=\left\{\begin{array}{cc}
\sum_{i=k}^{n} \sum_{j=k}^{i}\left(\begin{array}{l}
j \\
k
\end{array}\right)(-1)^{j-k} 2^{k}(2 j-k+1) a_{i}, & 0 \leq k \leq n \\
0 & , k>n
\end{array}\right.
$$

for all $k, n \in \mathbb{N}$. Therefore, we have by the relation (4.3) that

$$
\sum_{k=0}^{n} a_{k} x_{k}=\sum_{k=0}^{n} \sum_{i=k}^{n} \sum_{j=k}^{i}\left(\begin{array}{l}
j \\
k
\end{array}\right)(-1)^{j-k} 2^{k}(2 j-k+1) a_{i} y_{k}
$$


for all $n \in \mathbb{N}$. Then, (4.5) leads that $a x \in c s$ whenever $x \in \breve{c}$ if and only if $\widetilde{A} \in(c: c)$. This means that $a \in[\breve{c}]^{\beta}$ if and only if $\widetilde{A} \in(c: c)$. Hence, we have by Part (c) of Lemma 4.1 that the following conditions are satisfied:

$$
\begin{aligned}
& \sup _{n \in \mathbb{N}} \sum_{k=0}^{n}\left|\widetilde{a}_{n k}\right|=\sup _{n \in \mathbb{N}} \sum_{k=0}^{n}\left|\sum_{i=k}^{n} \sum_{j=k}^{i}\left(\begin{array}{l}
j \\
k
\end{array}\right)(-1)^{j-k} 2^{k}(2 j-k+1) a_{i}\right|<\infty, \\
& \exists\left(\alpha_{k}\right) \in \omega \text { such that } \lim _{n \rightarrow \infty} \widetilde{a}_{n k}=\lim _{n \rightarrow \infty} \sum_{i=k}^{n} \sum_{j=k}^{i}\left(\begin{array}{l}
j \\
k
\end{array}\right)(-1)^{j-k} 2^{k}(2 j-k+1) a_{i}=\alpha_{k} \\
& \exists \alpha \in \mathbb{C} \text { such that } \lim _{n \rightarrow \infty} \sum_{k=0}^{n} \widetilde{a}_{n k}=\lim _{n \rightarrow \infty} \sum_{k=0}^{n} \sum_{i=k}^{n} \sum_{j=k}^{i}\left(\begin{array}{l}
j \\
k
\end{array}\right)(-1)^{j-k} 2^{k}(2 j-k+1) a_{i}=\alpha
\end{aligned}
$$

which give that

$$
\left\{\sum_{k=0}^{n} \sum_{i=k}^{n} \sum_{j=k}^{i}\left(\begin{array}{l}
j \\
k
\end{array}\right)(-1)^{j-k} 2^{k}(2 j-k+1) a_{i}\right\}_{n \in \mathbb{N}} \in c .
$$

That is to say that the beta dual of the space $\breve{c}$ is the set $d_{2}$.

Theorem 4.4. $\left(\breve{c}_{0}\right)^{\gamma}=(\breve{c})^{\gamma}=\left(\breve{\ell}_{\infty}\right)^{\gamma}=d_{2}$, where the set $d_{2}$ is defined by (4.4).

Proof. This is similar to the proof of Theorem 4.3 with Part (b) instead of Part (c) of Lemma 4.1. So, we omit the detail.

\section{Matrix transformations related to the sequence space $\breve{c}$}

In this section, we characterize some matrix classes from the space $\breve{c}$ into the classical sequence spaces $\ell_{\infty}, c$ and $c_{0}$.

Theorem 5.1. $A=\left(a_{n k}\right) \in\left(\breve{c}: \ell_{\infty}\right)$ if and only if

$$
\begin{aligned}
& A_{n} \in \breve{c}^{\beta} \text { for each } n \in \mathbb{N}, \\
& \sup _{n \in \mathbb{N}} \sum_{k=0}^{\infty}\left|\sum_{i=k}^{\infty} \sum_{j=k}^{i}\left(\begin{array}{l}
j \\
k
\end{array}\right)(-1)^{j-k} 2^{k}(2 j-k+1) a_{n i}\right|<\infty .
\end{aligned}
$$

Proof. Suppose that $A=\left(a_{n k}\right) \in\left(\breve{c}: \ell_{\infty}\right)$ and $x=\left(x_{k}\right) \in \breve{c}$. Since $A x$ exists and belongs to the space $\ell_{\infty}$, the necessity of the condition (5.1) is obvious.

Consider the following equality derived from the $m^{t h}$ partial sum of the series $\sum_{k=0}^{\infty} a_{n k} x_{k}$ :

$$
\sum_{k=0}^{m} a_{n k} x_{k}=\sum_{k=0}^{m} \sum_{i=k}^{m} \sum_{j=k}^{i}\left(\begin{array}{l}
j \\
k
\end{array}\right)(-1)^{j-k} 2^{k}(2 j-k+1) a_{n i} y_{k}
$$

for all $m, n \in \mathbb{N}$. Therefore, by letting $m \rightarrow \infty$ in the equality (5.3) it is immediate that

$$
\sum_{k=0}^{\infty} a_{n k} x_{k}=\sum_{k=0}^{\infty} \sum_{i=k}^{\infty} \sum_{j=k}^{i}\left(\begin{array}{l}
j \\
k
\end{array}\right)(-1)^{j-k} 2^{k}(2 j-k+1) a_{n i} y_{k}
$$


for all $n \in \mathbb{N}$. Now, define the matrix $F=\left(f_{n k}\right)$ by

$$
f_{n k}:=\sum_{i=k}^{\infty} \sum_{j=k}^{i}\left(\begin{array}{l}
j \\
k
\end{array}\right)(-1)^{j-k} 2^{k}(2 j-k+1) a_{n i}
$$

for all $k, n \in \mathbb{N}$. Then, $A x=F y$ which gives that $F \in\left(c: \ell_{\infty}\right)$. Then, $F$ satisfies the condition (4.2) which is equivalent to (5.2).

Conversely, suppose that the conditions (5.1) and (5.2) hold, and take $x=\left(x_{k}\right) \in \breve{c}$. Then, (5.1) implies the existence of $A x$ and since the spaces $\breve{c}$ and $c$ are isomorphic we have $y \in c$. Therefore, (5.4) gives with (5.2) that

$$
\begin{aligned}
\|A x\|_{\infty} & =\sup _{n \in \mathbb{N}}\left|\sum_{k=0}^{\infty} a_{n k} x_{k}\right| \\
& \leq \sup _{n \in \mathbb{N}} \sum_{k=0}^{\infty}\left|\sum_{i=k}^{n} \sum_{j=k}^{i}\left(\begin{array}{l}
j \\
k
\end{array}\right)(-1)^{j-k} 2^{k}(2 j-k+1) a_{n i} y_{k}\right| \\
& \leq\|y\|_{\infty}\left[\sup _{n \in \mathbb{N}} \sum_{k=0}^{\infty}\left|\sum_{i=k}^{n} \sum_{j=k}^{i}\left(\begin{array}{l}
j \\
k
\end{array}\right)(-1)^{j-k} 2^{k}(2 j-k+1) a_{n i}\right|\right]<\infty .
\end{aligned}
$$

Hence, $A \in\left(\breve{c}: \ell_{\infty}\right)$.

This completes the proof.

Theorem 5.2. $A=\left(a_{n k}\right) \in(\breve{c}: c)$ if and only if the conditions (5.1) and (5.2) hold, and

$$
\begin{aligned}
& \exists \beta_{k} \in \mathbb{C} \text { such that } \lim _{n \rightarrow \infty} f_{n k}=\beta_{k} \text { for all } k \in \mathbb{N}, \\
& \exists \beta \in \mathbb{C} \text { such that } \lim _{n \rightarrow \infty} \sum_{k=0}^{\infty} f_{n k}=\beta,
\end{aligned}
$$

where $f_{n k}$ is defined by (5.5) as in the proof of Theorem 5.1.

Proof. Suppose that the conditions (5.1), (5.2), (5.6) and (5.7) hold, and take any $x=\left(x_{k}\right) \in \breve{c}$. The condition (5.1) implies the existence of $A$-transform of $x$. Then, one can derive by using the hypothesis (5.2) that

$$
\sum_{k=0}^{m}\left|\beta_{k}\right| \leq \sup _{n \in \mathbb{N}} \sum_{k=0}^{\infty}\left|f_{n k}\right|<\infty
$$

for all $m \in \mathbb{N}$. Hence, $\left(\beta_{k}\right) \in \ell_{1}$ which implies that $\left(\beta_{k} y_{k}\right) \in \ell_{1}$. Therefore, by taking in mind the relation (5.4) we derive for all $n \in \mathbb{N}$ that

$$
\sum_{k=0}^{\infty} a_{n k} x_{k}=\sum_{k=0}^{\infty}\left(f_{n k}-\beta_{k}\right) y_{k}+\sum_{k=0}^{\infty} \beta_{k} y_{k} .
$$

Then, we have by letting $n \rightarrow \infty$ that

$$
\lim _{n \rightarrow \infty}(A x)_{n}=\sum_{k=0}^{\infty} \beta_{k} y_{k} .
$$


Since $\left(\beta_{k} y_{k}\right) \in \ell_{1}$, (5.8) gives that $A x \in c$, that is, $A \in(\breve{c}: c)$.

Conversely, suppose that $A=\left(a_{n k}\right) \in(\breve{c}: c)$ and take $x \in \breve{c}$. Since the inclusion relation $c \subset \ell_{\infty}$ holds, the necessity of the conditions (5.1) and (5.2) follows from Theorem 5.1.

Now, consider the convergent sequences $u=\left(u_{k}\right)=\left\{u_{k}^{(n)}\right\}_{k \in \mathbb{N}}$ defined by (3.4) and $x=$ $\left(x_{k}\right)=\left\{\sum_{j=0}^{k}(-1)^{k-j} D_{k-j} T_{j}\right\}$. Since $A$-transforms of $u$ and $x$ exist, and belong to the space $c$ by the hypothesis, one can see that $A u=\left\{\sum_{j=k}^{\infty}(-1)^{j-k} D_{j-k} T_{k} a_{n j}\right\}_{n \in \mathbb{N}} \in c$ and $A x=$ $\left(\sum_{k=0}^{\infty} f_{n k}\right)_{n \in \mathbb{N}} \in c$ which show the necessity of the conditions (5.6) and (5.7), respectively.

This completes the proof.

Corollary 5.3. $A=\left(a_{n k}\right) \in\left(\breve{c}: c_{0}\right)$ if and only if (5.1), (5.2) hold, and (5.6) also holds with $\beta_{k}=0$ for all $k \in \mathbb{N}$.

Now, we can give the theorem characterizing the class of matrix transformations from a given sequence space $v$ to the Nörlund space $\lambda$.

Theorem 5.4. Suppose that $v$ be any given sequence space and the infinite matrices $A=\left(a_{n k}\right)$ and $\widetilde{D}=\left(\widetilde{d}_{n k}\right)$ are connected with the relation $\breve{d}_{n k}=\sum_{j=0}^{n} \sum_{i=0}^{n}\left(\begin{array}{c}n \\ i\end{array}\right) a_{i k} /\left[2^{n}(i+1)\right]$ for all $k, n \in \mathbb{N}$. Then, $A \in(v: \lambda)$ if and only if $\widetilde{D} \in(v: \mu)$.

Proof. Let $x=\left(x_{k}\right) \in v$. Consider the following equality

$$
\begin{aligned}
\sum_{k=0}^{m} \widetilde{d}_{n k} x_{k} & =\sum_{k=0}^{m} \sum_{j=0}^{n} \frac{1}{2^{n}} \sum_{i=0}^{n}\left(\begin{array}{c}
n \\
i
\end{array}\right) \frac{1}{i+1} a_{i k} x_{k} \\
& =\sum_{j=0}^{n}\left(\begin{array}{c}
n \\
j
\end{array}\right) \frac{1}{2^{n}} \sum_{i=0}^{n}\left(\begin{array}{c}
n \\
i
\end{array}\right) \frac{1}{i+1} \sum_{k=0}^{m} a_{i k} x_{k} \text { for all } m, n \in \mathbb{N} .
\end{aligned}
$$

Then, by letting $m \rightarrow \infty$ in (5.9) it is immediate that $(\widetilde{D} x)_{n}=\{\widetilde{B}(A x)\}_{n}$ for all $n \in \mathbb{N}$. Therefore, one can easily see that $\widetilde{D} x \in \mu$ if and only if $A x \in \lambda$. This completes the proof.

By combining Theorems 5.1, 5.2, 5.4 and Corollary 5.3, the following results are derived on the characterization of some matrix classes:

Corollary 5.5. Let $L=\left(l_{n k}\right)$ be an infinite matrix over the complex field. Then, the following statements hold:

(i) $L \in(\breve{c}: b s)$ if and only if (5.1) and (5.2) hold with $r_{n k}$ instead of $a_{n k}$, where $r_{n k}=\sum_{j=0}^{n} l_{j k}$ for all $k, n \in \mathbb{N}$.

(ii) $L \in\left(\breve{c}: b v_{\infty}\right)$ if and only if (5.1) and (5.2) hold with $r_{n k}$ instead of $a_{n k}$, where $r_{n k}=l_{n k}-$ $l_{n-1, k}$ for all $k, n \in \mathbb{N}$ and $b v_{\infty}$ denotes the space of all sequences $x=\left(x_{k}\right)$ such that $\left(x_{k}-\right.$ $\left.x_{k-1}\right) \in \ell_{\infty}$, (cf. Başar and Altay [4]). 
(iii) $L \in\left(\breve{c}: X_{\infty}\right)$ if and only if (5.1) and (5.2) hold with $r_{n k}$ instead of $a_{n k}$, where $r_{n k}=$ $\sum_{j=0}^{n} l_{j k} /(n+1)$ for all $k, n \in \mathbb{N}$ and $X_{\infty}$ denotes the space of all sequences $x=\left(x_{k}\right)$ such that $\left\{\sum_{k=0}^{n} x_{k} /(n+1)\right\} \in \ell_{\infty}$, (cf. Ng and Lee [26]).

(iv) $L \in\left(\breve{c}: r_{\infty}^{q}\right)$ if and only if (5.1) and (5.2) hold with $r_{n k}$ instead of $a_{n k}$, where $r_{n k}=\sum_{j=0}^{n}$ $q_{j} l_{j k} / Q_{n}$ for all $k, n \in \mathbb{N}$ and $r_{\infty}^{q}$ denotes the space of all sequences $x=\left(x_{k}\right)$ such that $\left(\sum_{k=0}^{n} q_{k} x_{k} / Q_{n}\right) \in \ell_{\infty}$, (cf. Altay and Başar [28]).

(v) $L \in\left(\breve{c}: a_{\infty}^{r}\right)$ if and only if (5.1) and (5.2) hold with $r_{n k}$ instead of $a_{n k}$, where $r_{n k}=\sum_{j=0}^{n}(1+$ $\left.r^{j}\right) l_{j k} /(1+n)$ for all $k, n \in \mathbb{N}$ and $a_{\infty}^{r}$ denotes the space of all sequences $x=\left(x_{k}\right)$ such that $\left\{\sum_{k=0}^{n}\left(1+r^{k}\right) x_{k} /(1+n)\right\} \in \ell_{\infty}$, (cf. Aydın and Başar [13]).

(vi) $L \in\left(\breve{c}: e_{\infty}^{r}\right)$ if and only if (5.1) and (5.2) hold with $r_{n k}$ instead of $a_{n k}$, where $r_{n k}=\sum_{j=0}^{n}$ $\left(\begin{array}{l}n \\ j\end{array}\right)(1-r)^{n-j} r^{j} l_{j k}$ for all $k, n \in \mathbb{N}$ and $e_{\infty}^{r}$ denotes the space of all sequences $x=\left(x_{k}\right)$ such that $\left\{\sum_{k=0}^{n}\left(\begin{array}{l}n \\ k\end{array}\right)(1-r)^{n-k} r^{k} x_{k}\right\} \in \ell_{\infty}$, (cf. Altay et al. [30]).

Corollary 5.6. Let $L=\left(l_{n k}\right)$ be an infinite matrix over the complex field. Then, the following statements hold:

(i) $L \in(\breve{c}: c s)$ if and only if (5.1), (5.2), (5.6) and (5.7) hold with $r_{n k}$ instead of $a_{n k}$, where $r_{n k}=\sum_{j=0}^{n} l_{j k}$ for all $k, n \in \mathbb{N}$.

(ii) $L \in(\breve{c}: c(\Delta))$ if and only if (5.1), (5.2), (5.6) and (5.7) hold with $r_{n k}$ instead of $a_{n k}$, where $r_{n k}=l_{n k}-l_{n-1, k}$ for all $k, n \in \mathbb{N}$ and $c(\Delta)$ denotes the space of all sequences $x=\left(x_{k}\right)$ such that $\left(x_{k}-x_{k-1}\right) \in c$, (cf. Başar [14]).

(iii) $L \in(\breve{c}: \widetilde{c})$ if and only if (5.1), (5.2), (5.6) and (5.7) hold with $r_{n k}$ instead of $a_{n k}$, where $r_{n k}=\sum_{j=0}^{n} l_{j k} /(n+1)$ for all $k, n \in \mathbb{N}$ and $\breve{c}$ denotes the space of all sequences $x=\left(x_{k}\right)$ such that $\left\{\sum_{k=0}^{n} x_{k} /(n+1)\right\} \in c$, (cf. Şengönül and Başar [27]).

(iv) $L \in\left(\breve{c}: r_{c}^{q}\right)$ if and only if (5.1), (5.2), (5.6) and (5.7) hold with $r_{n k}$ instead of $a_{n k}$, where $r_{n k}=\sum_{j=0}^{n} q_{j} l_{j k} / Q_{n}$ for all $k, n \in \mathbb{N}$ and $r_{c}^{q}$ denotes the space of all sequences $x=\left(x_{k}\right)$ such that $\left(\sum_{k=0}^{n} q_{k} x_{k} / Q_{n}\right) \in c$, (cf. Altay and Başar [28]).

(v) $L \in\left(\breve{c}: a_{c}^{r}\right)$ if and only if (5.1), (5.2), (5.6) and (5.7) hold with $r_{n k}$ instead of $a_{n k}$, where $r_{n k}=\sum_{j=0}^{n}\left(1+r^{j}\right) l_{j k} /(1+n)$ for all $k, n \in \mathbb{N}$ and $a_{c}^{r}$ denotes the space of all sequences $x=\left(x_{k}\right)$ such that $\left\{\sum_{k=0}^{n}\left(1+r^{k}\right) x_{k} /(1+n)\right\} \in c$, (cf. Aydın and Başar [29]).

(vi) $L \in\left(\breve{c}: e_{c}^{r}\right)$ if and only if (5.1), (5.2), (5.6) and (5.7) hold with $r_{n k}$ instead of $a_{n k}$, where $r_{n k}=\sum_{j=0}^{n}\left(\begin{array}{l}n \\ j\end{array}\right)(1-r)^{n-j} r^{j} l_{j k}$ for all $k, n \in \mathbb{N}$ and $e_{c}^{r}$ denotes the space of all sequences $x=\left(x_{k}\right)$ such that $\left\{\sum_{k=0}^{n}\left(\begin{array}{l}n \\ k\end{array}\right)(1-r)^{n-k} r^{k} x_{k}\right\} \in c$, (cf. Altay and Başar [31]).

Corollary 5.7. Let $L=\left(l_{n k}\right)$ be an infinite matrix over the complex field. Then, the following statements hold: 
(i) $L \in\left(\breve{c}: c s_{0}\right)$ if and only if the conditions (5.1), (5.2) and (5.6) of Corollary 5.3 hold with $r_{n k}$ instead of $a_{n k}$, where $c s_{0}$ denotes the space of all series converging to zero and $r_{n k}=$ $\sum_{j=0}^{n} l_{j k}$ for all $k, n \in \mathbb{N}$.

(ii) $L \in\left(\breve{c}: c_{0}(\Delta)\right)$ if and only if the conditions (5.1), (5.2) and (5.6) of Corollary 5.3 hold with $r_{n k}$ instead of $a_{n k}$, where $r_{n k}=l_{n k}-l_{n-1, k}$ for all $k, n \in \mathbb{N}$ and $c_{0}(\Delta)$ denotes the space of all sequences $x=\left(x_{k}\right)$ such that $\left(x_{k}-x_{k-1}\right) \in c_{0}$, (cf. Başar [14]).

(iii) $L \in\left(\breve{c}: \widetilde{c}_{0}\right)$ if and only if the conditions (5.1), (5.2) and (5.6) of Corollary 5.3 hold with $r_{n k}$ instead of $a_{n k}$, where $r_{n k}=\sum_{j=0}^{n} l_{j k} /(n+1)$ for all $k, n \in \mathbb{N}$ and $\breve{c}_{0}$ denotes the space of all sequences $x=\left(x_{k}\right)$ such that $\left\{\sum_{k=0}^{n} x_{k} /(n+1)\right\} \in c_{0}$, (cf. Şengönül and Başar [27]).

(iv) $L \in\left(\breve{c}: r_{0}^{q}\right)$ if and only if the conditions (5.1), (5.2) and (5.6) of Corollary 5.3 hold with $r_{n k}$ instead of $a_{n k}$, where $r_{n k}=\sum_{j=0}^{n} q_{j} l_{j k} / Q_{n}$ for all $k, n \in \mathbb{N}$ and $r_{0}^{q}$ denotes the space of all sequences $x=\left(x_{k}\right)$ such that $\left(\sum_{k=0}^{n} q_{k} x_{k} / Q_{n}\right) \in c_{0}$, (cf. Altay and Başar [28]).

(v) $L \in\left(\breve{c}: a_{0}^{r}\right)$ if and only if the conditions (5.1), (5.2) and (5.6) of Corollary 5.3 hold with $r_{n k}$ instead of $a_{n k}$, where $r_{n k}=\sum_{j=0}^{n}\left(1+r^{j}\right) l_{j k} /(1+n)$ for all $k, n \in \mathbb{N}$ and $a_{0}^{r}$ denotes the space of all sequences $x=\left(x_{k}\right)$ such that $\left\{\sum_{k=0}^{n}\left(1+r^{k}\right) x_{k} /(1+n)\right\} \in c_{0}$, (cf. Aydın and Başar [29]).

(vi) $L \in\left(\breve{c}: e_{0}^{r}\right)$ if and only if the conditions (5.1), (5.2) and (5.6) of Corollary 5.3 hold with $r_{n k}$ instead of $a_{n k}$, where $r_{n k}=\sum_{j=0}^{n}\left(\begin{array}{c}n \\ j\end{array}\right)(1-r)^{n-j} r^{j} l_{j k}$ for all $k, n \in \mathbb{N}$ and $e_{0}^{r}$ denotes the space of all sequences $x=\left(x_{k}\right)$ such that $\left\{\sum_{k=0}^{n}\left(\begin{array}{l}n \\ k\end{array}\right)(1-r)^{n-k} r^{k} x_{k}\right\} \in c_{0}$, (cf. Altay and Başar [31]).

\section{Conclusion}

To review the concerning literature about the domain of the infinite matrix $A$ in the sequence spaces $c_{0}, c$ and $\ell_{\infty}$, the following table may be useful: 
Table 5.1: The domain of some triangle matrices in the classical sequence spaces $c_{0}, c$ and $\ell_{\infty}$.

\begin{tabular}{|c|c|c|c|}
\hline$A$ & $\lambda$ & $\lambda_{A}$ & refer to: \\
\hline$\Delta$ & $c_{0}, c$ and $\ell_{\infty}$ & $c_{0}(\Delta), c(\Delta)$ and $\ell_{\infty}(\Delta)$ & {$[16]$} \\
$R^{t}$ & $c_{0}, c$ and $\ell_{\infty}$ & $r_{0}^{t}, r_{c}^{t}$ and $r_{\infty}^{t}$ & {$[17,28]$} \\
$B(r, s)$ & $c_{0}, c$ and $\ell_{\infty}$ & $\widehat{c}_{0}, \widehat{c}$ and $\widehat{\ell}_{\infty}$ & {$[18]$} \\
$B(r, s, t)$ & $c_{0}, c$ and $\ell_{\infty}$ & $c_{0}(B), c(B)$ and $\ell_{\infty}(B)$ & {$[19]$} \\
$C_{1}$ & $c_{0}, c$ and $\ell_{\infty}$ & $\widetilde{c}_{0}, \widetilde{c}$ and $X_{\infty}$ & {$[27,26]$} \\
$A^{r}$ & $c_{0}$ and $c$ & $a_{0}^{r}$ and $a_{c}^{r}$ & {$[29]$} \\
$E^{r}$ & $c_{0}, c$ and $\ell_{\infty}$ & $e_{0}^{r}, e_{c}^{r}$ and $e_{\infty}^{r}$ & {$[31,30]$} \\
$\Delta^{2}$ & $c_{0}$ and $c$ & $c_{0}\left(\Delta^{2}\right)$ and $c\left(\Delta^{2}\right)$ & {$[32]$} \\
$u \Delta^{2}$ & $c_{0}$ and $c$ & $c_{0}\left(u ; \Delta^{2}\right)$ and $c\left(u ; \Delta^{2}\right)$ & {$[33]$} \\
$\Delta^{m}$ & $c_{0}$ and $c$ & $c_{0}\left(\Delta^{m}\right)$ and $c\left(\Delta^{m}\right)$ & {$[34,35]$} \\
$R^{q}$ & $c_{0}$ and $c$ & $(\bar{N}, q)_{0}$ and $(\bar{N}, q)$ & {$[36]$} \\
$\Delta^{(m)}$ & $c_{0}$ and $c$ & $c_{0}\left(\Delta^{(m)}\right)$ and $c\left(\Delta^{(m)}\right)$ & {$[37]$} \\
$G(u, v)$ & $c_{0}, c$ and $\ell_{\infty}$ & $c_{0}(u, v), c(u, v)$ and $\ell_{\infty}(u, v)$ & {$[38]$} \\
$\Lambda$ & $c_{0}$ and $c$ & $c_{0}^{\lambda}$ and $c^{\lambda}$ & {$[39]$} \\
$B(\widetilde{r}, \widetilde{s})$ & $c_{0}, c$ and $\ell_{\infty}$ & $\widetilde{c}_{0}, \widetilde{c}$ and $\widetilde{\ell}_{\infty}$ & {$[40]$} \\
$A_{\lambda}$ & $c_{0}$ and $c$ & $A_{\lambda}\left(c_{0}\right)$ and $A_{\lambda}(c)$ & {$[41]$} \\
$\widehat{F}$ & $c_{0}$ and $c$ & $c_{0}(\widehat{F})$ and $c(\widehat{F})$ & {$[42]$} \\
$N^{t}$ & $c_{0}, c$ and $\ell_{\infty}$ & $c_{0}\left(N^{t}\right), c\left(N^{t}\right)$ and $X_{a(p)}$ & {$[43,44]$} \\
\hline
\end{tabular}

In 1978, the domain of Cesàro matrix $C_{1}$ of order one in the classical sequence spaces $\ell_{\infty}$ and $\ell_{p}$ were introduced by $\mathrm{Ng}$ and Lee [26], where $1 \leq p<\infty$. Following Ng and Lee [26], Şengönül and Başar [27] have studied the domain of Cesàro matrix $C_{1}$ of order one in the classical sequence spaces $c_{0}$ and $c$. In 1978, the domain of Nörlund matrix $N^{t}$ in the classical sequence spaces $\ell_{\infty}$ and $\ell_{p}$ were introduced by Wang [44], where $1 \leq p<\infty$. Quite recently, Başar [12] has investigated the domain $\tilde{\ell}_{p}$ of the matrix $\widetilde{B}$ in the space $\ell_{p}$ with $1 \leq p<\infty$. As a natural continuation of Başar [12], we have worked on the domains $\breve{\ell}_{\infty}, \breve{c}$ and $\breve{c}_{0}$ of the matrix $\widetilde{B}$ in the classical sequence spaces $\ell_{\infty}, c$ and $c_{0}$.

Although the matrix transformations from the domain of certain triangles in the classical sequence spaces into the classical sequence spaces have been characterized, the matrix transformations from the domain of Nörlund matrix in the space of convergent sequences into some classical sequence spaces have not been characterized, until now. So, combining Theorems 5.1, 5.2, 5.4 and Corollary 5.3 has a special importance for this type studies, in future.

Finally, we note that in spite of the domain $\widetilde{\ell}_{p}$ of the composite matrix $\widetilde{B}$ in the space $\ell_{p}$ have investigated by Başar [12] in the case $1 \leq p<\infty$, investigation of the space $\tilde{\ell}_{p}$ remains open in the case $0<p<1$. 


\section{Acknowledgement}

We would like to thank Professor Eberhard Malkowsky for his valuable remarks on the earlier version of this paper which improved the presentation and readability.

\section{References}

[1] E. Malkowsky, E. Savaş, Matrix transformations between sequence spaces of generalized weighted mean, Appl. Math. Comput. 147 (2004), 333-345.

[2] M. Candan, Almost convergence and double sequential band matrix, Acta Math. Sci., Ser. B, Engl. Ed., 34 (2014), 354-366.

[3] K. Kayaduman and M. Şengönül, The spaces of Cesàro almost convergent sequences and core theorems, Acta Math. Sci. Ser. B Engl. Ed., 32 (2012), 2265-2278.

[4] B. Altay, F. Başar, The fine spectrum and the matrix domain of the difference operator $\Delta$ on the sequence space $\ell_{p},(0<p<1)$, Commun. Math. Anal. 2 (2007), 1-11.

[5] R. Çolak, M. Et, E. Malkowsky, Some Topics of Sequence Spaces, in: Lecture Notes in Mathematics, Fırat Univ. Press (2004), 1-63, ISBN: 975-394-0386-6.

[6] B. Choudhary and S. K. Mishra, A note on Köthe-Toeplitz duals of certain sequence spaces and their matrix transformations, Internat. J. Math. Math. Sci., 18 (1995), 681-688.

[7] M. A. Sarıgöl, On difference sequence spaces, J. Karadeniz Tech. Univ. Fac. Arts Sci. Ser. Math. -Phys., 10 (1987), $63-71$.

[8] S. K. Mishra, Matrix maps involving certain sequence spaces, Indian J. Pure Appl. Math., 24 (1993), $125-132$.

[9] A. K. Gaur and M. Mursaleen, Difference sequence spaces, Int. J. Math. Math. Sci., 21 (1998), 701-706.

[10] Ç. A. Bektaş, M. Et and R. Çolak, Generalized difference sequence spaces and their dual spaces, J. Math. Anal. Appl., 292 (2004), 423-432.

[11] A. Sönmez, Almost convergence and triple band matrix, Math Comput. Model., 57 (2013), $2393-2402$.

[12] F. Başar, Domain of the composition of some triangles in the space of p-summable sequences, AIP Conference Proceedings, 1611 (2014), 348-356.

[13] C. Aydın, F. Başar, Some new sequence spaces which include the spaces $\ell_{p}$ and $\ell_{\infty}$, Demonstratio Math., 38 (2005), 641-656.

[14] F. Başar, Summability Theory and Its Applications, Bentham Science Publishers, e-books, Monographs, İstanbul, 2012.

[15] E. Malkowsky and V. Rakočević, On matrix domains of triangle, Appl. Math. Comput., 189 (2007), $1146-1163$.

[16] H. Kizmaz, On certain sequence spaces, Canad. Math. Bull., 24 (1981), 169-176.

[17] F. Başar, B. Altay, On the space of sequences of p-bounded variation and related matrix mappings, Ukrainian Math. J., 55 (2003), 136-147.

[18] M. Kirişçi and F. Başar, Some new sequence spaces derived by the domain of generalized difference matrix, Comput. Math. Appl., 60 (2010), 1299-1309.

[19] A. Sönmez, Some new sequence spaces derived by the domain of the triple band matrix, Comput. Math. Appl., 62 (2011), 641-650.

[20] P. K. Kamthan and M. Gupta, Sequence Spaces and Series, Marcel Dekker Inc., New York and Basel, 1981.

[21] A. Wilansky, Summability through Functional Analysis, North-Holland Mathematics Studies 85, Amsterdam - Newyork • Oxford, 1984.

[22] A. M. Jarrah and E. Malkowsky, BK spaces, bases and linear operators, Rendiconti Circ. Mat. Palermo II, 52 (1990), 177-191.

[23] R.G. Cooke, Infinite Matrices and Sequence Spaces, Macmillan and Co. Limited, London, 1950.

[24] I. J. Maddox, Elements of Functional Analysis, The University Press, Cambridge, second edition, 1988. 
[25] M. Stieglitz, H. Tietz, Matrix transformationen von folgenraumen eine ergebnisübersict, Math. Z., 154 (1977), $1-16$.

[26] P.-N. Ng and P.-Y. Lee, Cesàro sequence spaces of non-absolute type, Comment. Math. Prace Mat., 20 (1978), 429-433.

[27] M. Şengönül and F. Başar, Some new Cesàro sequence spaces of non-absolute type which include the spaces $c_{0}$ and c, Soochow J. Math., 31 (2005), 107-119.

[28] B. Altay and F. Başar, Some paranormed Riesz sequence spaces of non-absolute type, Southeast Asian Bull. Math., 30 (2006), 591-608.

[29] C. Aydın and F. Başar, On the new sequence spaces which include the spaces $c_{0}$ and c, Hokkaido Math. J., 33 (2004), 383-398.

[30] B. Altay, F. Başar and M. Mursaleen, On the Euler sequence spaces which include the spaces $\ell_{p}$ and $\ell_{\infty} I$, Inform. Sci., 176 (2006), 1450-1462.

[31] B. Altay and F. Başar, Some Euler sequence spaces of non-absolute type, Ukrainian Math. J., 57 (2005), 1-17.

[32] M. Et, On some difference sequence spaces, Turkish J. Math., 17 (1993), 18-24.

[33] M. Mursaleen, Generalized spaces of difference sequences, J. Math. Anal. Appl., 203 (1996), 738-745.

[34] M. Et and R. Çolak, On some generalized difference sequence spaces, Soochow J. Math., 21 (1995), 377-386.

[35] R. Çolak and M. Et, On some generalized difference sequence spaces and related matrix transformations, Hokkaido Math. J., 26 (1997), 483-492.

[36] E. Malkowsky, Recent results in the theory of matrix transformations in the sequence spaces, Mat. Vesnik, 49 (1997), 187-196.

[37] E. Malkowsky and S. D. Parashar, Matrix transformations in space of bounded and convergent difference sequences of order $m$, Analysis, 17 (1997), 87-97.

[38] B. Altay nad F. Başar, Some paranormed sequence spaces of non-absolute type derived by weighted mean, J. Math. Anal. Appl., 319 (2) (2006), 494-508.

[39] M. Mursaleen and A. K. Noman, On the spaces of $\lambda$-convergent and bounded sequences, Thai J. Math., 8 (2010), 311-329.

[40] M. Candan, Domain of the double sequential band matrix in the classical sequence spaces, J. Inequal. Appl., 2012, 2012:281, 15 pp.

[41] N. L. Braha and F. Başar, On the domain of the triangle $A(\lambda)$ on the spaces of null, convergent and bounded sequences, Abstr. Appl. Anal., 2013, Article ID 476363, 9 pages, 2013, doi:10.1155/2013/476363.

[42] M. Başarır, F. Başar and E. E. Kara, On the spaces of Fibonacci difference null and convergent sequences, Sarajevo J. Math., Vol.12 (25), No.2, (2016), 1-16.

[43] O. Tuğ and F. Başar, On the domain of Nörlund mean in the spaces of null and convergent sequences, TWMS J. Pure Appl. Math., 7 (2016), 76-87.

[44] C. S. Wang, On Nörlund sequence space, Tamkang J. Math., 9 (1978), 269-274.

Kısıklı Mah. Alim Sok. Alim Apt. No: 7/6, 34692 - Üsküdar/İstanbul, Turkey.

E-mail: fbasar@fatih.edu.tr; feyzibasar@gmail.com

Department of Mathematics and Computer Sciences, University of Prishtina, Avenue "Mother Teresa", No=5, Prishtine, 10000, Kosova.

E-mail: nbraha@yahoo.com 